\title{
Kongressberichte
}

\section{Kongress der ECLSO (European Contact Lens Society of Ophthalmologists)}

27. bis 29. September 2002 in Bordeaux

Im Vorjahr war in der Versammlung der Nationalrepräsentanten der ECLSO in Santander/Spanien beschlossen worden, auch in Bordeaux am Tag vor dem eigentlichen Kongress einen Aus- bzw. Fortbildungstag für das Spezialgebiet „Kontaktologie" zu veranstalten. Dies sollte unter dem Motto „Augenärzte für Augenärzte" erfolgen. So wurde diese Fortbildung am 26. September 2002 unter der Leitung von Florence Malet (Bordeaux) im Hôspital universitaire Pellegrin abgehalten. In zwei Blöcken hielten verschiedene Experten Vorträge über Basiswissen (vormittags) und Spezialgebiete (nachmittags). Alle Vorträge waren in englischer Sprache und fanden das Interesse vieler - besonders junger Kollegen/innen. Der Hörsaal war sehr gut besucht. Sicher wird diese Einfühung in den nächsten Jahren fortgeführt werden.

Die wesentlichen Themen der zahlreichen Vorträge waren „Trockenes Auge und Kontaktlinsen“, Versorgung von Kindern mit Kontaktlinsen, Sport, Kosmetik und Kontaktlinsen,
Allergie und Kontaktlinsen und Keratokonus. Neuen Materialien zum Langzeittragen von Kontaktlinsen waren ebenfalls einige Referate gewidmet. Eine große Industrieausstellung gab einen guten Überblick über das heutige Angebot. Das heurige Gala-Dinner fand - wie in dieser Gegend nicht anders zu erwarten - in einem Château eines Weingutes statt.

Für die (leider) wenigen österreichischen Teilnehmer bot sich eine interessante Gelegenheit, in Vorträgen und auch persönlichen Gesprächen die Probleme der augenärztlichen Kontaktologie in anderen europäischen Ländern kennenzulernen. Der verbindende Geist der ECLSO war deutlich zu spüren.

Die Zusammensetzung des neuen Präsidiums finden Sie unter ,Gesellschaftsberichte" in diesem Heft.

Der 33. Kongress wird von 18. bis 21. September 2003 in Venezia - Mestre, stattfinden.

Information:

Homepage: www.eclso2003.sistemacongressi.com, E-Mail: mannucci@ux1.unipd.it

Herbert Schuster, ECLSO - Nationalrepräsentant Österreichs Kontakt und Mitgliederaufnahme:

E-Mail: schuster@augenkontakt.at

\section{Herbst-Symposium der Vereinigung kontakt- linsenanpassender Augenärzte}

Am 12. Oktober 2002 fand traditionellerweise in Salzburg das Herbst-Symposium der Vereinigung kontaktlinsenanpassender Augenärzte statt. Trotz einer gleichzeitig stattfindenden Glaukomveranstaltung in der Steiermark war die Tagung sehr gut besucht, im Vortragsraum blieb - praktisch bis zum Schluss - kein Sitzplatz leer. Die heurigen Vorträge waren bunt gemischt: Prof. Roth (Ulm/Washington D.C.) befasste sich in zwei Referaten mit "Kontaktlinsenkomplikationen" und - nachmittags - mit ethischen und juristischen Aspekten der Kontaktoptik. Mag. Peter Pirkner (Wien) widmete seinen sehr lebendigen Vortrag der „Stressbewältigung in der täglichen Praxis" (dieses Thema behandelte er auch im nachmittags abgehaltenen Seminar für Ordinationsassistentinnen). Zwei Vorträge (Frau Prof. Eva Haller-Schober und Dr. Klaus Hiti - beide Univ.-Augenklinik Graz) befassten sich mit „Labordiagnostischen Maßnahmen rund um die Kontaktlin$\mathrm{se}^{\text {" }}$ und „Akanthamöbenbefall und KL-Desinfektion"; beide Themen sind infolge von Pressemitteilungen vor kurzer Zeit in der Öffentlichkeit aktuell. Dr. Kurt-Peter Polzhofer bot eine umfassende Übersicht über Kontaktlinsenmaterialien und deren Entwicklung bis zum heutigen Stand - Basiswis- sen für Kontaktologen. In der Reihe „Aus der Praxis - für die Praxis "wurden die Themen „Multifocal-Kontaktlinsen“ von Dr. Thomas Pfleger (Wien) und ,Führerscheingutachten“ von Dr. Karl Riedler (Wels) behandelt. Viele Zuhörer benutzten die „Kontaktologische Fragestunde“ am späten Nachmittag zur Diskussion über aktuelle medizinische und berufspolitische Probleme.

Die Kaffeepausen sowie das gemeinsame Mittagessen boten einerseits Gelegenheit zum Besuch der großen Industrieausstellung, andererseits zur Pflege interkollegialer Kontakte.

Im Rahmen des Symposiums wurde die jährliche Generalversammlung abgehalten, das Ergebnis der Wahl des neuen Vorstandes wird in der Rubrik "Gesellschaftsberichte" bekanntgegeben.

Das 8. Herbst-Symposium wird am 11 . Oktober 2003 in Salzburg stattfinden.

Anfragen und Vormerkung für Programmversand:

Sekretariat der Vereinigung kontaktlinsenanpassender Augenärzte, Ungargasse 27, A-1030 Wien, Fax: 01/713 1715 14, Homepage: www.augenkontakt.at, E-Mail: linsen-info@,augenkontakt.at H. Schuster, Wien 